\title{
Infection and Cancer Screening in Potential Living Donors: Best Practices to Protect the Donor and Recipient
}

\author{
Varvara A. Kirchner • Patty T Liu • Timothy L. Pruett
}

Published online: 20 January 2015

(C) Springer International Publishing AG 2015

\begin{abstract}
Twenty-one percent of transplants in the US come from live donors. Data show that live-donor transplantation is a safe practice, but is not completely risk free. Malignancy and infection transmission through live organ donation is extremely rare, but can be a devastating event for the recipient, donor and treatment team if it occurs. The donor evaluation is multifactorial, taking into the account the unique medical, social and family history of individual donors, needs of the recipient, and determination of the anatomic and functional suitability of the donor organ, and is further complicated by geographical and temporal components. While balancing all practical considerations can be complex, a thorough medical assessment for infection and malignancy of potential living donor is central in protecting the donor and the intended transplant recipient.
\end{abstract}

Keywords Living donor screening - Infection .

Malignancy · Transplant

\section{Introduction}

Live organ donation plays an integral role as a potential source of organs for transplantation with no significant numerical

This article is part of the Topical Collection on Live Kidney Donation

V. A. Kirchner • P. T Liu $\cdot$ T. L. Pruett $(\varangle)$

Department of Surgery, Division of Transplantation, University of

Minnesota, 420 Delaware St SE, MMC 195,

Minneapolis, MN 55455, USA

e-mail: tlpruett@umn.edu

V. A. Kirchner

e-mail: kirc0079@umn.edu

P. T Liu

e-mail: liux2757@umn.edu cap. In the US, living donors comprised $42 \%(n=5,990)$ of the entire donor pool for 2013, supplying the organs for $21 \%$ of transplants performed (http://optn.transplant.hrsa.gov). The intent of the live donation procedure is to remove an organ from a person (the donor) for the benefit of another (the recipient), with as few adverse consequences to either as feasible.

The live-donor evaluation is therefore divided into two important and distinct elements. First, the potential donor must be screened for conditions that will impact his/her mortality and morbidity risks during and after the organ donation procedure; the conclusions of this assessment should be clearly reflected in the consent prior to proceeding with donation. Second, the donor's organ must be assessed to ensure that the recipient will receive adequate function, that the organ is anatomically feasible to implant and that the risk of disease transmission through the organ to the recipient is small.

The Organ Procurement and Transplantation Network $(\mathrm{OPTN}) /$ United Network for Organ Sharing (UNOS) has developed policy requirements that address the evaluation of organs from live and deceased donors and the mandatory reporting of potential disease transmissions after an organ transplant (http://optn.transplant.hrsa.gov/media/1140/ policy_notice_12-2014.pdf). Reports made through the patient safety portal about potential disease transmissions are much less frequent after live, in contrast to deceased, organ transplantation. In 2013, 12 potential disease transmissions (of 5,990 live donations) were reported to the Disease Transmission Advisory Committee (DTAC), and none were judged to have been a proven/probable disease transmission (personal communication, Sarah Taranto, UNOS research). While the full potential of disease transmission after livedonor organ transplant is probably not entirely recognized, the risk appears to be significantly less than after transplant from a deceased organ donor. From 2008-2013, the DTAC received only 32 cases of potential live-donation disease 
transmission (the majority are malignancy with only one proved/probable transmission), while getting over 1,000 reports from deceased donors.

\section{Risk}

Live organ donation has a legacy of providing excellent, safe organs for transplantation. Assessment for diseases that can affect the recipient has always been a part of the donor evaluation process. However, a specific numerical risk cannot be definitively generated and practitioners must piece together supporting information from other sources. This review is intended to give some of the information surrounding the donor evaluation.

\section{Pertinent Donor History: Risk Assessment}

A fundamental premise for the process of live donation and transplantation is the individual's autonomy to decide whether to give or receive an organ. Both donor and recipient should be provided information about the organ and pertinent details about the other individual (donor/recipient) that may affect their risk/benefit analysis and personal decision to proceed with the donation and/or transplantation. The potential donor must know that an assessment for transmissible diseases is an essential component of the evaluation and any such information that is important for the recipient decision to accept will be shared. If there is information that the potential donor is reluctant to share, the decision to proceed with evaluation should be reconsidered. The disclosure issue is becoming more complex with increasing numbers of non-directed, paired exchange and chain donations, where donor/recipient pairs have no close relationship. The policy specifics of what is required to be disclosed during the informed consent are not clearly delineated as different individuals need variable information in their decision-making processes. As part of the consent, the possibility that the donor evaluation testing may uncover a new diagnosis with future medical or social implications including a potential mandatory reporting of the diagnosis to the public health agencies or to the recipient in order to proceed with the donation should be disclosed. Transplant programs must clearly address these complex medical issues in the informed consent process. Judicious use of the Independent Donor Advocate can be a valuable asset in helping to determine that individual donors understand and are satisfied with the information presented to them.

An important element of the donor evaluation is to detect diseases that the potential donor may be unaware of or may not consider relevant to the donation process. Inquiry about prior diagnoses of bacterial, fungal and viral infections including their sequelae such as hepatitis should be pursued.
Confirmed or suspected malignancies including mole removals and the age-appropriate cancer screening history are important for donor and recipient risk stratification prior to donation. Additionally, the family and social history give insight into potential exposures to diseases such as tuberculosis, malaria, travel to or immigration from endemic areas (Strongyloides, coccidiodomycosis, Chagas disease, etc.) and inquiry for genetic predisposition to malignancies.

\section{Malignancy}

Fortunately, malignancy transmission through living donor organs has proven to be very infrequent. However, an ageappropriate cancer risk evaluation thorough examination and family history are warranted as elements for donor and recipient risk stratification. Despite all the emphasis upon infection transmission, malignancy has been the most common unexpected live-donor potentially derived disease transmission event (PDDTE) reported to the DTAC. From 2008 to 2013, malignancy accounted for $27 \%$ of the total PDDTEs reported among deceased donors (DDs), whereas for live donors (LDs) malignancy accounted for $69 \%$ (Fig. 1) (personal communication, Sarah Taranto, UNOS research). The majority of potential transmission events were reported after the donation when a prior organ donor was diagnosed with malignancy. Only one organ obtained from a live donor has been discerned by the DTAC to be a proved/probable transmission of a

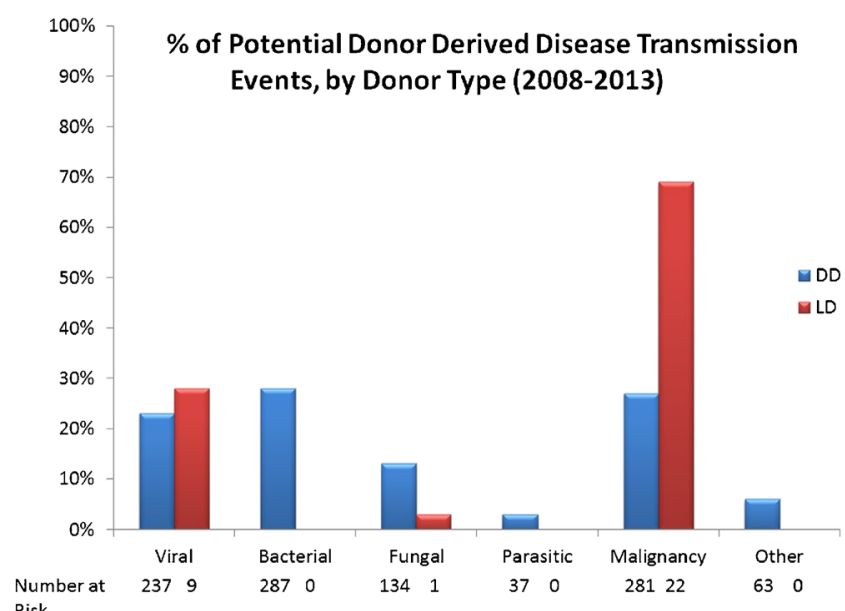

Fig. 1 Percent of potential donor-derived disease transmission events between 2008 and 2013 by donor type and type of event reported to the OPTN and reviewed by the DTAC. The $X$-axis indicates the number of potential transmission events by the donor type and type of event. One out of $22(5 \%)$ malignancy PDDTEs from live donors resulted in a proven/ probable transmission. All other reports were not associated with disease transmission. Nine viral PDDTEs in live donors included HBV, HCV, HTLV, WNV and HSV. There were no disease-derived attributable deaths among live-donor recipients 
malignancy to the recipient. This experience reinforces the differences between live and deceased organ donors, in that biologic processes continue to occur in live organ donors after medical evaluation and subsequently after donation.

In 2011, the DTAC Malignancy Subcommittee published six risk categories for donor tumor transmission, assigning a specific risk category to the individual tumor types and including recommendations on the clinical use of the organs based on these risk categories [ $[1]$. Stratification of tumors into different risk categories was based on individual literature reports, cancer registry review and OPTN registry data. This publication did not differentiate between deceased and living donor tumor transmission, but provided a framework based on best available data to guide clinical decision making regarding the suitability of potential donors with a history of malignancy and need of specific screening to identify donors with a high risk of tumor transmission. No significant risk category was defined as benign tumor where malignancy has been excluded. The minimal risk category included tumors with 0 to $0.01 \%$ of transmission events per organ transplant from donors with specific tumors; these tumors included nonmelanoma skin cancers, noninvasive carcinoma of the bladder (for nonrenal transplants only), small papillary or follicular carcinoma of the thyroid and solitary welldifferentiated $(\leq 1 \mathrm{~cm})$ renal cell carcinoma. The low-risk category (0.1-1 \% of transmission events) included small renal cell carcinoma $(1-2.5 \mathrm{~cm})$, low-grade CNS tumors, primary CNS mature teratoma, solitary papillary thyroid carcinoma $(0.5-2.0 \mathrm{~cm})$, minimally invasive follicular carcinoma (1.0$2.0 \mathrm{~cm})$ and a history of treated non-CNS malignancy $\left(\nabla_{-}\right.$ 5 years prior) with $>99 \%$ probability of cure. The intermediate risk category (1-10\% of transmission events) included breast and colon carcinoma in situ, resected welldifferentiated renal cell carcinoma $(4-7 \mathrm{~cm})$ and a history of treated non-CNS malignancy ( $\geq 5$ years prior) with a probability of cure between 90-99\%. The high-risk category was defined by $>10 \%$ of transmission events and comprised of a current or past history of melanoma, leukemia/lymphoma or neuroendocrine tumors; breast or colon cancer $>$ stage 1 ; choriocarcinoma; any CNS tumor with ventriculoperitoneal or ventriculoarterial shunt, metastasis or high-grade (III/IV) histology; any treated non-CNS malignancy with insufficient follow-up to predict behavior, incurable or with $<90 \%$ probability of cure; metastatic carcinoma; sarcoma; stage I-IV lung cancer; renal cell carcinoma $>7 \mathrm{~cm}$ or any other active cancer. The last category proposed by the DTAC was tumors of unknown risk category. This review suggested that donors in the no significant risk category are considered standard; minimalrisk category organs can be used based on clinical judgment with informed consent of the recipient; low-risk category organs could be considered for recipients at significant risk of mortality without transplant and with the detailed informed consent $[1 \bullet]$.
In general, any active malignancy in the potential living donor is considered to be a contraindication to the donation, as organ donation is secondary to health maintenance of the potential donor. However, there have been reports of living donor renal transplants from patients with $<3 \mathrm{~cm}$ RCC that were excised on the back table with subsequent successful transplant. In one study, out of 43 transplants performed (5 DD and 38 LD), there was only one recurrence of RCC within a transplanted kidney 9 years after the original operation $[2$, 3]. Another group reported their living donor transplant experience from eight donors with resected RCC and eight donors with resected low-grade ureteral cancer; the group reported no recurrences of RCC and a single recurrence of ureteral cancer 15 months after the transplant $[3,4]$. While the risk of RCC transmission appears to be low after resection, the unknown but additional risk should be reflected in the informed consent process.

The risk of malignancy transmission will vary depending on whether the potential transplanted organ harbors the malignancy versus the organ is coming from the donor with a malignancy from a different organ of origin. The risk assessment in this situation should be made by a multidisciplinary team including oncologic consultation. Even a remote history of certain malignancies among potential living donors is considered to be an absolute contraindication to donation; these include melanoma, choriocarcinoma, hematological malignancy, monoclonal gammopathy, and testicular, lung and breast cancer [5•]. Although mostly based on the deceased donor experience, choriocarcinoma and melanoma carry very high transmission rates (93\% and $74 \%$, respectively) and mortality among recipients (64\% and $58 \%$ ) [6]. Lung cancer and hematologic malignancies also carry high transmission rates and in previous reports were associated with high rates of death among recipients, whereas donors with a history of breast cancer are avoided because of the potential late metastasis and aggressive biology of the tumor [5•, 7].

In summary, most of the current knowledge on malignancy transmission from living donors is extrapolated from deceased donor data. There were few reports including breast and lung carcinoma transmission through the living donation [8]. However, given the younger age of most living donors, in contrast to deceased donors, and thorough preoperative assessment prior to the donation, the event of malignancy transmission through a donation is extremely rare [9]. At the same time, long-term follow-up post-donation may reveal a new diagnosis of malignancy in the donor, which may affect the management of the transplant recipient in the future.

\section{Cancer Screening/Family History}

Common cancers in the general population should be specifically considered among live donors, and appropriate 
screening should be performed prior to the donation based on guidelines for the jurisdiction where the donation will occur. In the US, the American Cancer Society (ACS) screening guidelines for cervical, breast, prostate, colon, skin and lung cancer should be followed $[10 \cdot 11 \bullet]$. The most frequently reported transmission of non-CNS tumor has been renal cell carcinoma; however, this report is primarily based on deceased donor experience [12]. In light of the thorough livedonor evaluation and modern imaging, it is unlikely that renal cell carcinoma would remain undetectable on preoperative evaluations. On the other hand, a history of skin malignancy or mole removal in the distant past may be overlooked despite detailed assessment of the potential living donor.

Furthermore, attention should be given to the recipients with end-stage disease secondary to specific etiologies such as bilateral renal cell carcinoma, who could have a familial predisposition. Therefore, even with normal imaging of a living related donor prior to the donation, donor-derived de novo renal cell carcinoma could be diagnosed in recipients in the future. Other familial syndromes affecting the kidney, liver or pancreas should be considered in the process of living donor evaluation. The transplant team should recognize that the malignancy risk increases with increased age of the donor as well as that the incidence of certain malignancies may vary among countries. The fact that some malignancies will go undetected prior to donation should be taken into the consideration; therefore, live donors must be monitored post donation for a new diagnosis of malignancy.

\section{Infection Testing}

Policy and Rationale Knowledge of preexisting infection/ disease of a donor allows for more complete assessment of donor safety and morbidity from the donation procedure for rational planning of pre- and post-transplantation recipient care and for possible donor exclusion if the risk to the recipient is high. There has been concern about the lack of standardization of testing; therefore, the OPTN policy stipulates that laboratories performing tests for organ donation must meet national accreditation standards and use test kits that have been approved by the FDA to detect specific pathogens.

The OPTN live-donor evaluation policy requires that the live organ donor be screened for all pathogens required for deceased organ donation, including CMV, EBV, HIV, HBV, $\mathrm{HCV}$ as well as other possible geographic and/or seasonal pathogens. A unique aspect for live organ donors is that the initial donor testing may occur significantly before the time of donation, in contrast to deceased donation, when testing is performed at the time of death and organ retrieval. In fact, this time difference was an important issue for a recipient who developed HIV post-kidney transplant from a live donor. The donor had tested HIV negative 2 months prior to donation. This case brought into focus that live organ donors continue to have environmental exposures before and after a specific testing event [13]. The consequence of this transmission is behind the recommendation in the revised PHS guidelines that HIV, HBV and HCV testing be performed as close to the donation as possible, but certainly within 1 month of the donation event. It was also recommended that donors with increased risk behaviors be counseled about avoidance of risky behaviors prior to donation.

Much of the current interest in disease transmission through organ transplantation is the result of public discussion of HIV/HCV transmission to organ recipients. Criteria for "increased risk" donors have been part of the safety assessment for organ and tissue donation. After the transmission of HIV and HCV from a deceased organ donor to four transplant recipients in 2007 [14], the Secretary of Health and Human Services (HHS) directed the OPTN to generate and enforce a policy stating that an individual receiving an organ from an "increased risk" donor (defined by the 1994 PHS guidelines) must be informed and willing to accept such an organ. In 2013, the Public Health Services (PHS) revised the guidelines specific for organ donors; "increased risk" donors are now defined as those with one or more risk factors for HIV, HCV or HBV infection. The OPTN policy stipulates that all potential (living and deceased) donors be assessed for the increased behavioral risks identified by the PHS guidelines. The presence of each risk factor indicates an increased risk of all three pathogens as there is an overlap of associated risk; the 11 risk factors are defined in Table 1 [15].

It is incumbent that US centers incorporate questions about sexual activity, drug use, incarceration time and recent infectious exposures in order to identify the donors deemed by the PHS as being at increased risk for HIV, HBV and HCV and document that the recipients have been informed about the donor risk, irrespective of donor testing results.

$H I V$ The National Organ Transplant Act (1984) precluded the use of organs from a donor infected with the HIV virus. Medical advancements in HIV therapy have led many to query the relevance of this exclusion, and the law has recently been amended to allow the use of organs from HIV+ donors under yet to be defined research restrictions. However, it should be feasible for an HIV+ individual to be a live organ donor in the future. Multiple tests have been developed over the years to detect the virus with variable levels of sensitivity and reliability. Tests to detect HIV include direct detection of viral nucleic acid (PCR/NAT), HIV antigen p24 or antibodies generated against HIV antigens. The oldest and most commonly used test has been the detection of antibodies directed against HIV antigens (anti-HIV+). This has been the only mandated test by OPTN policy to determine whether a donor is HIV infected. A positive test would stop the evaluation process for organ donation. However, it can take up to 3 weeks 
Table 1 Factors identified in the literature to be associated with increased likelihood of recent HIV, HBV or HCV infection

People who have had sex with a person known or suspected to have HIV, $\mathrm{HBV}$ or HCV infection in the preceding 12 months

Men who have had sex with men (MSM) in the preceding 12 months

Women who have had sex with a man with a history of MSM behavior in the preceding 12 months

People who have had sex in exchange for money or drugs in the preceding 12 months

People who have had sex with a person who had sex in exchange for money or drugs in the preceding 12 months

People who have had sex with a person who injected drugs by the intravenous, intramuscular or subcutaneous route for nonmedical reasons in the preceding 12 months

A child who is $<18$ months of age and born to a mother known to be infected with or at increased risk for HIV, HBV or HCV infection

A child who has been breastfed within the preceding 12 months and the mother is known to be infected with or at increased risk for HIV infection

People who have injected drugs by the intravenous, intramuscular or subcutaneous route for non-medical reasons in the preceding 12 months

People who have been in lockup, jail, prison or a juvenile correctional facility for more than 72 consecutive $h$ in the preceding 12 months

People who have been newly diagnosed with or have been treated for syphilis, gonorrhea, Chlamydia or genital ulcers in the preceding 12 months

(With permission from Seem DL, et al. Excerpt from the PHS Guideline for Reducing HIV, HBV and HCV Transmission Through Organ Transplantation. Am J Transplant. 2013; 13:1953-1962) [15]

after virus infection to generate an antibody response, and HIV has been transmitted from organs obtained during the "window period." To decrease the possibility that a recent, undetected HIV infection has occurred, NAT (detection within 5-6 days of infection) or antigen/antibody combination tests (positive within 7-14 days after infection) have been recommended by the PHS guidelines for individuals with increased risk [16]. It is highly likely that this recommendation will be adopted as national policy.

$H B V$ This DNA virus is globally ubiquitous with disparate prevalence rates in people from different geographies. Infection with $\mathrm{HBV}$ is most commonly controlled by the immune response, but can cause a chronic infection/hepatitis in a percentage of infected people. The commonly used tests to detect active HBV infection are viral products, HBsAg and HBV-DNA. HBsAg is probably the more relevant test, as it remains detectable even when viral replication is suppressed by either immunity or antiviral medications. Immune response to prior HBV exposure is typically detected by antibody generation to structural HBV antigens, $\mathrm{HBs} A b$ and $\mathrm{HBcAb}$ (antibody reacting with surface and core proteins). Detection of prior HBV (contained) infection is important predominantly for liver donors. Since HBV is a DNA virus, persistence of viral genetic material is common in its dominant reservoir, i.e., the liver. Liver recipients of $\mathrm{HBcAb}+$ livers have a significant risk for developing active $\mathrm{HBV}$ infection after transplantation in the absence of antiviral therapies. Recipients of non-hepatic organs from an $\mathrm{HBcAb}+, \mathrm{HBsAg}$ - donor appear to have little risk of acquiring active HBV infection [17]. OPTN policy states that $\mathrm{HBsAg}$ and $\mathrm{HBcAb}$ should be performed on organ donors to assess the risk of HBV transmission. Active HBV infection $(\mathrm{HBsAg}+)$ is usually a reason to exclude live organ donation because of the potential increased risk to the donor and recipient. Latent $\mathrm{HBV}$ infection $(\mathrm{HBcAb}+)$ in the donor must be weighed as a risk for all liver recipients, but is much less of an issue in kidney recipients. HBV-DNA+, in the absence of $\mathrm{HBsAg}$, is a relative risk of uncertain significance, so the infectious potential in this setting is unknown. While lowlevel HBV-DNA is theoretically infectious to an organ recipient, the frequency of the infection appears to be very low in either the deceased or living donor pool.

$\mathrm{HCV}$ All organ donors must be tested for infection with HCV, an RNA virus that is a common cause of chronic infection and hepatitis. HCV has been transmitted to naïve organ recipients through live and deceased organs as well as through the use of isolated vascular grafts from infected deceased donors [14, 18]. As with HIV, multiple tests are available to discern the presence of the virus. The most common test has been the detection of antibodies generated against structural and nonstructural viral proteins. In the time prior to routine clinical use of polymerase chain reactions (PCRs) for the detection of viral RNA, a variety of tests for antibody detection to specific viral proteins was developed for clinical use. About $15 \%$ of people with anti-HCV antibodies will not have detectable HCV-RNA in the serum. The new PHS guidelines stipulate that all donors should be tested using HCV-RNA PCR. Similar to HIV, antiviral therapies can make viral RNA undetectable in the blood, so antibody testing should not be omitted as a screening test. However, to be consistent with PHS guidelines, the final test assessed during the month prior to live donation should be HCV-RNA PCR (NAT).

$C M V$ and $E B V$ The herpes viruses are common within the general population and testing is typically used to discern the risk for recipient disease and use/length of chemoprophylaxis. The presence of anti-CMV or anti-EBV (anti-viral capsular antigen or anti-Epstein Barr nuclear antigen) signifies prior donor infection, with the potential that the latent virus will reactivate and cause infection, particularly in the virusnaïve recipient. While it is rare that detection of these viruses will preclude donation, knowing that an organ comes with a latent virus is an important factor in post-transplant recipient care [19]. The most commonly used tool for prior infection is the detection of antibodies directed against viral proteins. Rarely, there may be the suspicion that a potential donor is 
experiencing an acute $\mathrm{CMV} / \mathrm{EBV}$ infection, where detection of viral nucleic acid in the serum or blood may be assessed.

Bacteria Chronic or recurrent bacterial infections typically exclude an individual from donation. Acute symptomatic disease is also a reason to postpone the donation event. However, detection of asymptomatic bacteriuria is not infrequent, especially in female donors. While there are no randomized studies confirming the safety of transplanting these kidneys, the literature is relatively silent about adverse sequelae. Donor testing for syphilis is mandated by the OPTN policy although there have been no reported cases of disease transmission from transplantation of organs from the infected deceased or live donors.

\section{Special Cases}

TB/Geography/Seasonality There are a variety of diseases that have a markedly heterogeneous prevalence based on the geography, season or both. While the transmission risk of tuberculosis is quite low from the asymptomatic, previously exposed but untreated individual [positive purified protein derivative (PPD) test or interferon gold assay], anecdotal transmission reports warrant donor testing for those at risk of a past exposure [20]. Decisions to treat the donor before or after the donation should be made for the health of the individual, irrespective of recipient status. The risk to the organ recipient is low from an untreated latent TB donor, but most should be treated.

Seasonal predilection for mosquito-borne diseases, such as West Nile Virus (WNV) in the US with $90 \%$ of cases diagnosed between July-September, should alert the donor team if a potential donor from regions of the country with disease prevalence has had a viral syndrome associated with headache [21]. However, $80 \%$ of immunocompetent individuals will be asymptomatic carriers of WNV infection, but the likelihood of symptomatic infection with central nervous system involvement is 40 times greater in immunosuppressed patients according to some sources [22]. Several reviews describe cases of transplant-related WNV transmissions involving severe neurologic sequelae, permanent damage and death in transplant recipients [23]. Since there is a lag period between the original live-donor testing and the time of donation, re-testing of potential donors closer to the time of the donation should be performed based on the provider's clinical judgment.

While there have been no rabies virus transmissions through live-donor transplantation, the most recent case of raccoon rabies infection in the US was unusual as the transmission was not recognized until 18 months after the transplantation from a deceased donor who had not had a history of a recent exposure [24]. Thus, questions regarding animal bites or travel history may facilitate identification of past exposures [22]. HTLV-1/2 are enveloped RNA viruses with $65 \%$ of homology resulting in chronic infection; only HTLV-1 has been implicated in the development of adult T-cell lymphoma/leukemia (ATL) and progressive spastic paralysis [22]. The endemic regions for the infection include southwestern Japan, the Caribbean, sub-Saharan Africa, part of South America, Middle East and Oceania. A dozen cases of live and deceased donor transmission of HTLV-1 have been reported in the world, but up until recently none had been reported in the US. In 2013, the first US transmission of HTLV-1 that led to HTLV-associated myelopathy (HAM) in the recipient was documented from a live donor, native to the Dominican Republic. This transmission exemplifies the importance of geographic recognition and subsequent selective donor testing in certain situations [25]. Another relevant geographic pathogen that is endemic to the US southwest desert is coccidiodomycosis. Prophylactic administration of antifungal agents to recipients of live and deceased donor organs from the southwest US has become commonplace. Coccidioides disease primarily causes pulmonary infection; however, more than half of immunocompetent subjects are asymptomatic. Transplant recipients with transmitted disease typically develop disseminated disease affecting all organ systems [22]. Serological screening for coccidioidomycosis in donors and recipients from endemic areas may help identify populations at risk, but no clear evidence is available to recommend universal application. One retrospective review highlighted four live seropositive donor cases without pre-donation prophylaxis that did not result in coccidioidomycosis infection among recipients [26]. Histoplasma is ubiquitous in the Ohio River valley of the US. Histoplasmosis infection among transplant patients is predominantly thought to be a de novo disease, although there have been two cases of transmission attributed to organ transplantation. Serologic testing is infrequent and controversial. There is still controversy about prophylactic itraconazole use in recipients of solid organs from seropositive donors as the etiology of infection is less likely to be from organ transmission in contrast to environmental exposures.

A plethora of other geographic diseases have been transmitted through deceased donors, including but not limited to malaria, strongyloides, Chagas disease, echinococcosis, and filariae- and trematodes-associated infections; of those, there have already been a few documented instances of transmission or there is a theoretical risk of transmission through a live donation. Unfortunately, there are no conclusive data that allow for testing and inclusion/exclusion for organ donors. A recent report of Chagas-naïve recipients transplanted with kidneys from Chagas + organ donors without a recognized disease transmission reiterates the uncertainties of the risk and need/lack of strategy for recipient management [27]. While many tropical diseases have been recognized as possibly being transmitted to organ recipients and with an increasing 


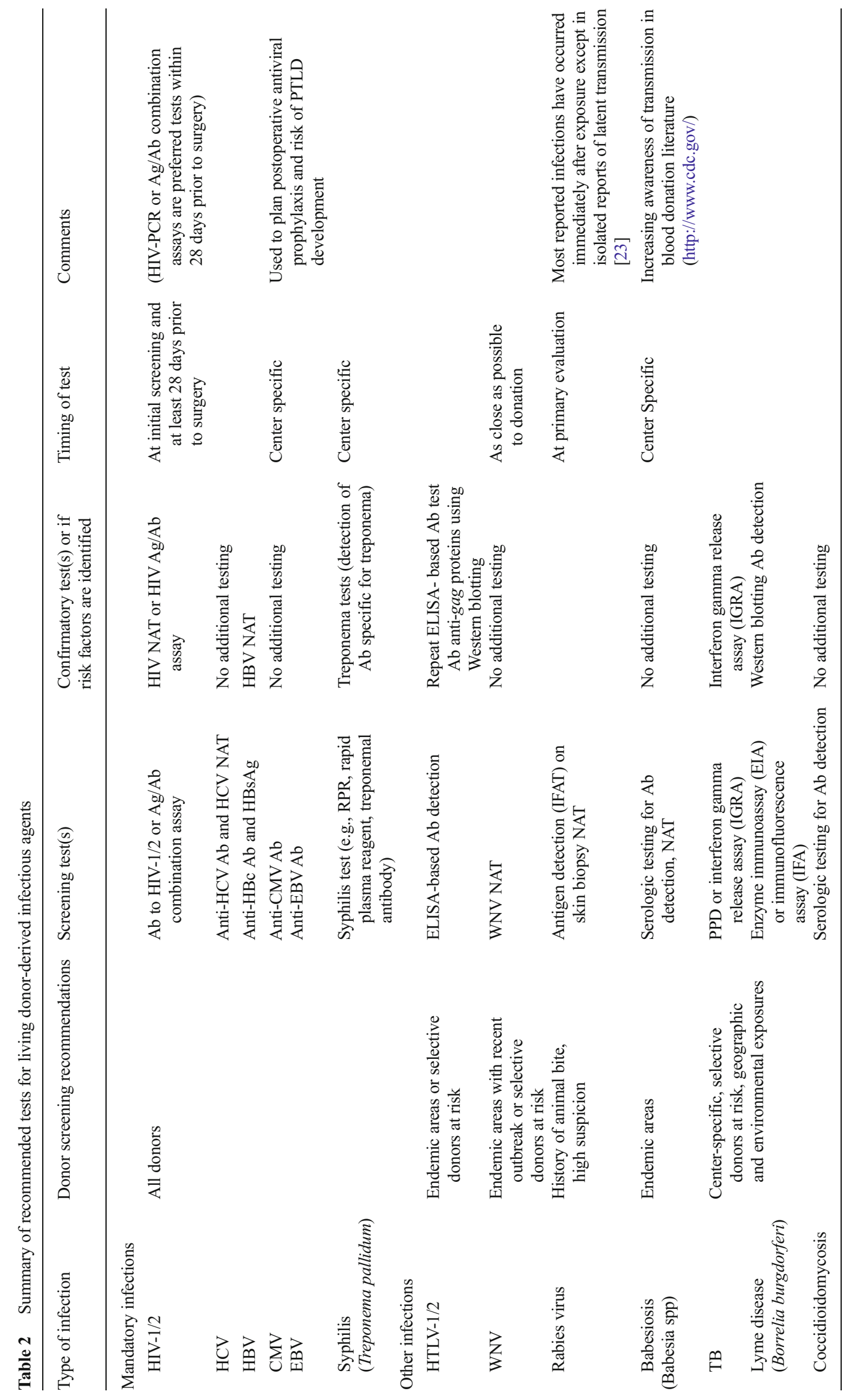


immigrant population in the US, there is still a paucity of clinical data to state conclusively how/when to test a donor. The authors feel however that as the consequences of disease transmission can be life threatening, donors from endemic areas with a significant potential risk of exposure to Chagas disease and strongyloides should be assessed, and any positive results should be communicated to the potential recipient. Optimal management strategies are not known and may include avoidance of the transplant, donor treatment, recipient prophylaxis, or recipient surveillance and treatment. This recommendation may inadvertently exclude a few potential organ donors with little risk for disease transmission, but with case reports of morbidity and mortality associated with transmission and availability of serologic and tissue testing, we feel that it is prudent to test donors from endemic areas.

SOT recipients being infected with other pathogens, such as Leishmania, barbesiosis, amebiasis, cysticerocsis and trypanosomiasis, have been described, but the definitive evidence of transmission and risk have not been documented [22]. Geographic and seasonal diseases are changing in the US, and in the blood donation field, babesiosis is being recognized as an increasing threat in New England [28]. However, many of the above-mentioned infections have a very low prevalence in the US; the judicious testing of live donors based on a donor's medical and social history, place of origin, travel history and clinical suspicion is recommended. Engagement of an infectious disease expert with knowledge of geographic medicine is encouraged for clarification of risks and appropriate testing.

\section{Conclusions}

In the modern transplant practice, live-donor transplantation is a safe alternative to deceased donor transplantation, but it is not risk free for either the donor or recipient. Assessment of the live organ donor is a dual-purpose process. The primary goal is to ascertain any underlying medical issues that would add undue risk to the organ donor. However, there is also a duty to the recipient to provide an organ with known risks. Some risks are manageable and have a favorable risk/benefit relationship; at the same time, informed risk stratification is important in order to minimize the known risks and most importantly to highlight unacceptable risks, thus avoiding unexpected outcomes after the transplantation.

National policy requirements and clinical practice guidelines provide a backbone for live-donor evaluation; however, each living donor is unique and requires additional considerations based on his/her medical, social and family history. Recent events in deceased organ donors (rabies, West Nile virus, balamuthia) considerably influenced the live organ donor workup and emphasized the importance of environmental exposure of the donor; however, given the very low prevalence of these infections and absence of widely available testing for some of these pathogens (e.g., balamuthia serology), universal donor screening would not be practical [22-24, 29]. Furthermore, in the case of live donation, the time period between testing and donation should be taken into consideration as the transmissible disease may be contracted by a donor during this period. Only the performance of HIV, HCV and HBV testing, as close as possible but within 28 days of donation, is mandated in the US; the timing of other infectious workups remains center-specific. Table 2 provides a summary of infectious agents and recommendations that the transplant team should be aware of at the time of donor evaluation (http://optn.transplant.hrsa.gov/media/1140/policy_notice 12-2014.pdf) [22] (http://www.cdc.gov/). Although both infection and malignancy transmissions have occurred through live organ donation, in the global view, the number of these events is negligible in contrast to the successful lifesaving transplants performed each year. However, since the risk of the transmission is not zero, good medical practice requires consistent donor evaluation and reasonable follow-up so that our care can be improved using good medical information.

\section{Compliance with Ethics Guidelines}

Conflict of Interest Varvara A. Kirchner, Patty Liu and Timothy L. Pruett declare that they have no conflict of interest.

Human and Animal Rights and Informed Consent This article does not contain any studies with human or animal subjects performed by any of the authors.

\section{References}

Papers of particular interest, published recently, have been highlighted as:

- Of importance

1. Nalesnik MA et al. Donor-transmitted malignancies in organ transplantation: assessment of clinical risk. Am J Transplant. 2011;11(6): 1140-7. Assessment of donor clinical risk in malignancy transmission.

2. Nicol DL et al. Kidneys from patients with small renal tumors: a novel source of kidneys for transplantation. BJU Int. 2008;102(2): 188-92.

3. Mannami M et al. Last resort for renal transplant recipients, 'restored kidneys' from living donors/patients. Am J Transplant. 2008;8(4):811-8.

4. Khurram MA, et al. Renal transplantation with kidneys affected by tumors. Int J of Neph. 2011: 6 .

5. Zhang S, Yuan J, Li W, Qifa Y. Organ Transplantation from donors (cadaveric or living) with a history of malignancy: review of the 
literature. Transplant Rev. 2014;28:169-75. Review of malignancy transmission through organ transplantation.

6. Buell J et al. Donor transmitted malignancies. Ann Transplant. 2004;9(1):53-6.

7. Ison MG et al. Donor-derived disease transmission events in the United States: data reviewed by the OPTN/UNOS Disease Transmission Advisory Committee. Am J Transplant. 2009;9(8):1929-35.

8. Kaufmann M, et al. Transplant tumor registry: donor related malignancies. 2002; 74(3):358-62.

9. Chapman JR, Webster AC, Wong G. Cancer in the transplant recipient. Cold Spring Harb Perspect Med. 2013;3(7).

10. http://www.cancer.org/ (Provides Current Cancer Screening Guidelines)

11. OPTN Policies. 2014. Living Donation Policy 14: $168 \mathrm{http} / / /$ optn. transplant.hrsa.gov/governance/policies/. OPTN policy on medical evaluation of living donor.

12. Gandhi MJ, Strong DM. Donor derived malignancy following transplantation. Cell Tissue Bank. 2007;8(4):267-86.

13. Echenique IA et al. Impact of repeat testing of living kidney donors within 14 days of the transplant procedure: a multicenter retrospective survey. Transpl Infect Dis. 2014;16(3):403-11.

14. Ison $\mathrm{MG}$ et al. Transmission of human immunodeficiency virus and hepatitis $\mathrm{C}$ virus from an organ donor to four transplant recipients. Am J Transplant. 2011;11(6):1218-25.

15. Seem DL et al. Excerpt from PHS Guideline for Reducing HIV, HBV and HCV Transmission Through Organ Transplantation. Am J Transplant. 2013;13:1953-62.

16. Ison MG et al. Donor-derived infections in solid organ transplantation. Am J Transplant. 2013;13(s4):22-30.

17. Feng $\mathrm{S}$ et al. Organ donors with positive viral serology or malignancy: risk of disease transmission by transplantation. Transplantation. 2002;74(12):1657-63.

18. Ison MG, Nalesnik MA. An update on donor-derived disease transmission in organ transplantation. Am J Transplant. 2011;11(6): $1123-30$.
19. Hodson EM et al. Antiviral medications to prevent cytomegalovirus disease and early death in recipients if solid-organ transplants: a systematic review of randomized controlled trials. Lancet. 2005;365:2105-15.

20. Rose D. The risk of tuberculosis transmission in solid organ transplantation: is it more than a theoretical concern? Can J Infect Dis Med Microbiol. 2005;16(5):304-8.

21. Lindsey NP et al. West Nile Virus and other arboviral diseasesUnited States, 2013. MMWR. 2014;63(24):521-6.

22. Martin-Davila P et al. Transmission of Tropical and geographically restricted infections during solid-organ transplantation. Clin Microbiol Rev. 2008;21(1):60-96.

23. Greenwald MA, Kuehnert MJ, Fishman JA. Infectious disease transmission during organ and tissue transplantation. Emerg Infect Dis. 2012;18(8)

24. Vora NM et al. Raccoon rabies virus variant transmission through solid organ transplantation. JAMA. 2013;310(4):398-407.

25. Ramanan $\mathrm{P}$ et al. Donor transmitted HTLV-1-associated myelopathy in a kidney transplant recipient-case report and literature review. Am J Transplant. 2014;14(10):2417-21.

26. Blair JE, Mulligan DC. Coccidioidomycosis in healthy persons evaluated for liver or kidney donation. Transpl Infect Dis. 2007;9(1):78-82.

27. Cicora $\mathrm{F}$ et al. Use of kidneys from Trypanosoma Cruzi-infected donors in naïve transplant recipients without prophylactic therapy: the experience in a high-risk area. Transplantation. 2014;97(1):e34.

28. Johnson ST et al. Seroprevalence of Babesia microti in blood donors from babesia-endemic areas of the northeastern United States: 2000 through 2007. Transfusion. 2009;49(12):2574-82.

29. Gupte AA et al. Transmission of Balamuthia mandrillaris through solid organ transplantation: Utility of organ recipient serology to guide clinical management. Am J Transplant. 2014;14(6):1417-24. 